\title{
Anomalous transfer in rats: A "macropattern" phenomenon
}

\author{
P. C. DODWELL, R. R. NIEMI, and H. B. FERGUSON \\ Queen's University, Kingston, Ontario, Canada
}

\begin{abstract}
Anomalous transfer (AT) is defined in the immediately preceding paper (Dodwell, Ferguson, \& Niemi, 1975). The object of the first two experiments reported here was to find out what role, if any, the pattern elements of the AT training stimuli play in generating the effect. It was found that patterns made up with different elements, such as triangles or circles rather than squares, still yield AT, and in fact patterns in similar orientations made up of different sorts of elements are indiscriminable from one another. The third experiment demonstrates, in line with the interpretation of AT as a transposition phenomenon along a horizontality-verticality continuum, that when only one orientation is available as a basis for discrimination between a pair of patterns, the discrimination is extremely difficult for rats, and perhaps impossible.
\end{abstract}

Anomalous transfer (AT) was defined in the immediately preceding paper (Dodwell, Ferguson, \& Niemi, 1975) as preference in a transfer test which directly contradicts the training contingencies of a conventional two-choice discrimination task. That is, certain novel stimulus displays are treated as "more positive" or "more negative" than the positive and negative training patterns, respectively. It was shown in the same paper that AT occurs for a class of similar pattern pairs, in which certain pattern parameters vary, so long as "horizontality" and "verticality" are separately maintained in the training pairs as well as in the transfer pairs. The types of pattern used are shown as Figure 1 in Dodwell et al. (1975).

The phenomenon of AT has been demonstrated in several species, but always with basically the same types of pattern array. A question of interest is whether or not the phenomenon is a "macropattern" effect. If it turned out that the pattern elements (the small squares which constitute the parts of the pattern) are important in generating $\mathrm{AT}$, this would cast some doubt on the interpretation offered by Dodwell (1970). This interpretation was in terms of summation of activity in cortical cells having either predominantly vertically oriented, or horizontally oriented, retinal receptive fields. If different "micropatterns" (pattern elements) led to different effects, some other explanation for AT would be required. The following two experiments examine the question of whether micropattern discrimination occurs, or is relevant.

This research was supported by Grant AOA44 from the National Research Council of Canada to the first author. R. R. Niemi is now at the University of Manitoba, Winnipeg, Manitoba. H. B. Ferguson is now at Carleton University, Ottawa, Ontario.

\section{EXPERIMENT I}

The object of this experiment was to find out whether rats can discriminate between different pattern elements of the sort used in studies of AT. Failure to discriminate would strengthen the argument that $\mathrm{AT}$ is a macropattern phenomenon.

\begin{abstract}
Method
Subjects and apparatus. The subjects were 10 male Sprague-Dawley rats, about 3 months old at the start of training. They were run in the same apparatus as the subjects in Dodwell et al. (1975).

Stimuli. The training stimuli were those of Figure 1 (above) in Dodwell et al. (1975). Transfer patterns consisted of columns at a 3:1 center-to-center ratio, whose pattern elements consisted of squares, triangles, or circles, all of equal area.

Procedure. Shaping, pretraining, and training on rows and columns of squares were the same as in Experiment I of Dodwell et al. (1975). Subsequently, the subjects were divided randomly into two groups. Group A received training for 50 trials per day on columns of squares and columns of triangles, Group B on circles and triangles. In both cases, training continued to a criterion of $90 \%$ on 1 day, or for 500 trials. Transfer tests were planned but turned out to be inappropriate.
\end{abstract}

\section{Results and Discussion}

In no case was the discrimination learned. Table 1 shows the mean performance over the 10 days for each group, together with the range of scores on each day. Clearly there is no case where a subject approaches criterion performance, although one animal in Group A reached $70 \%$ or better on two occasions. Overall, its choice of squares was about $60 \%$. It is reasonable to conclude that, in general, the pattern elements are below the rat's threshold for detail vision in this experimental situation.

\section{EXPERIMENT I}

Having shown that micropattern discriminations 
Table 1

Percentage Choice on Transfer Tests: Experiment I

\begin{tabular}{|c|c|c|c|c|c|c|c|c|c|c|}
\hline \multicolumn{11}{|c|}{ Squares (+) vs. Triangles } \\
\hline $\begin{array}{l}\text { Mean Percentage } \\
\text { Range } \\
\text { Day }\end{array}$ & $\begin{array}{c}56 \\
44-66 \\
1\end{array}$ & $\begin{array}{c}48 \\
38-64 \\
2\end{array}$ & $\begin{array}{c}61 \\
50-74 \\
3\end{array}$ & $\begin{array}{c}53 \\
46-62 \\
4\end{array}$ & $\begin{array}{c}56 \\
52-66 \\
5\end{array}$ & $\begin{array}{c}53 \\
48-60 \\
6\end{array}$ & $\begin{array}{c}55 \\
40-64 \\
7\end{array}$ & $\begin{array}{c}50 \\
42-58 \\
8\end{array}$ & $\begin{array}{c}52 \\
48-58 \\
9\end{array}$ & $\begin{array}{c}55 \\
46-70 \\
10\end{array}$ \\
\hline \multicolumn{11}{|c|}{ Circles $(+)$ vs. Triangles } \\
\hline $\begin{array}{l}\text { Mean Percentage } \\
\text { Range } \\
\text { Day }\end{array}$ & $\begin{array}{c}56 \\
38-62 \\
1\end{array}$ & $\begin{array}{c}52 \\
32-62 \\
2\end{array}$ & $\begin{array}{c}48 \\
46-50 \\
3\end{array}$ & $\begin{array}{c}50 \\
46-56 \\
4\end{array}$ & $\begin{array}{c}52 \\
42-58 \\
5\end{array}$ & $\begin{array}{c}44 \\
34-52 \\
6\end{array}$ & $\begin{array}{c}45 \\
34-55 \\
7\end{array}$ & $\begin{array}{c}49 \\
44-60 \\
8\end{array}$ & $\begin{array}{c}46 \\
36-50 \\
9\end{array}$ & $\begin{array}{c}53 \\
48-56 \\
10\end{array}$ \\
\hline
\end{tabular}

are difficult, and perhaps impossible, it seems unlikely that changing the pattern elements will affect $\mathrm{AT}$ in any way.

Nevertheless, an experiment was run to check on the possibility. Since the experiment turned out exactly as expected, and in fact simply constitutes another replication of AT, it is not reported in detail.

The experiment was run using eight rats, in exactly the same paradigm as has previously been used to demonstrate AT. The patterns were like those in Figure 1 of Dodwell et al. (1975), except that the pattern elements consisted of circles or triangles (base horizontal), the elements being of the same area as the squares used previously. Four animals were trained with circle patterns, four with triangle patterns, and all showed AT to both positive and negative stimuli when presented with smooth stripes in conjunction with the appropriate training patterns. This further strengthens the view that AT is a macropattern phenomenon and that the nature of the pattern elements is not important.

The results of these two experiments support the argument for the continuum hypothesis, i.e., that AT is a transposition of discriminations along a "horizontality-verticality" dimension. A different way to add further weight to the hypothesis would be to show that, with arrays of the same sort as those used in demonstration of AT, discrimination fails if only one dimension, say horizontality, is available as a discriminandum.

\section{EXPERIMENT III}

The object of this experiment was to discover whether rats can discriminate between horizontal separations in patterns which contain a constant amount of vertical contour. More precisely, the aim was to find out whether summation of contour information along the principal visual axes, of the sort held to underlie AT, occurs in a direction orthogonal to the main axis of pattern orientation. The patterns shown in Figure 1 were used. The argument has been that summation occurs in row and column figures because the pattern elements stimulate units with both horizontal and vertical receptive fields, but do so differentially (Dodwell, 1970). Smooth stripes, however, are optimum stimuli for units with receptive fields in their orientation, so it might be that no summation would occur in the orthogonal orientation (horizontally, for the patterns of Figure 1). Alternatively, one could argue that if horizontal receptive fields are large enough to cover the whole horizontal extent of the patterns of Figure 1, summation on that dimension would be equal for both of them. In either case, the differences in relative horizontal separation of contours in the two patterns would not provide a discriminable cue. The amount of vertical contour is the same in the two patterns, so one can predict that discrimination between them should be very difficult, if not impossible.

\section{Method}

Apparatus and training procedure were the same as in Experiment I, the patterns shown in Figure 1 being used. Eight male rats similar to those used before were trained to a criterion of $90 \%$ correct on one day, or to 500 trials. Transfer tests were planned, but proved to be inappropriate. The obvious parallel discrimination, using the patterns rotated through $90 \mathrm{deg}$, was not run in view of the rat's well-known propensity for discriminating only the bottoms of patterns if an obvious differential cue (in this case, brightness) is available.

\section{Results and Discussion}

No single subject reached criterion, and overall both the group and individual data clustered around a probability of $50 \%$ correct. The data are summarized in Table 2.

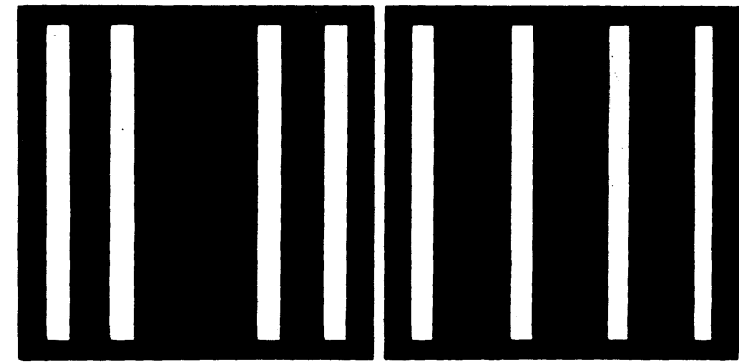

Figure 1. Shapes used in Experiment III to demonstrate failure of discrimination when horizontal separation and vertical stripes is the only cue available. 
Table 2

Percentage Choice on Original Training: Experiment III

\begin{tabular}{|c|c|c|c|c|c|c|c|c|c|c|}
\hline $\begin{array}{l}\text { Mean Percentage } \\
\text { Range } \\
\text { Day }\end{array}$ & $\begin{array}{c}51 \\
47-58 \\
1\end{array}$ & $\begin{array}{c}54 \\
45-62 \\
2\end{array}$ & $\begin{array}{c}55 \\
46-65 \\
3\end{array}$ & $\begin{array}{c}54 \\
50-58 \\
4\end{array}$ & $\begin{array}{c}54 \\
52-57 \\
5\end{array}$ & $\begin{array}{c}50 \\
41-54 \\
6\end{array}$ & $\begin{array}{c}56 \\
52-62 \\
7\end{array}$ & $\begin{array}{c}49 \\
44-51 \\
8\end{array}$ & $\begin{array}{c}50 \\
47-52 \\
9\end{array}$ & $\begin{array}{c}54 \\
46-57 \\
10\end{array}$ \\
\hline
\end{tabular}

The experiment was replicated, with similar results. It appears that discrimination. of horizontal separation per se, in the absence of different amounts of vertical contour, is a difficult problem for the rat. This result is quite consistent with the findings of Niemi and Dodwell (1971), but in that case each discriminandum contained both horizontal and vertical contours. At all events, the present finding reinforces the opinion that responding to the patterns used in the AT paradigm is based on summation of contour information along the most salient orientation in a given pattern, and that contour separation by itself is not a readily used cue.

\section{CONCLUSION}

The experiments reported here and in the immediately preceding paper (Dodwell,. Ferguson, \& Niemi, 1975) support, in the main, the original interpretation of AT as a relational discrimination phenomenon, in which relative amounts of summation along two dimensions which are mutually perpendicular is the critical factor. The fact that changing the absolute amounts of summation (or center-to-center ratios of pattern elements) has little effect so long as the relative amounts remain in the right direction, and also that AT is a macropattern phenomenon, are strong evidence for that interpretation.

The latency data reported in the first paper are disappointing, in that they turn out to be unusable for scaling discrimination difficulty, at least using our procedure, but are informative in indicating that rats can probably discriminate the difference between stripes and interrupted stripes, even though in certain cases they treat them as equivalent.

All patterns used in these experiments preserved an overall square configuration (with some minor variations being necessary in order to achieve the various center-to-center ratios) and were equated for brightness and brightness gradients such as "brighter-above" and "brighter-below." It is thus reasonable to conclude that the discriminations obtained were genuine pattern discriminations and not based on some more primitive set of cues.

It is never possible, of course, to say that discriminations not achieved in a particular experiment are impossible; another apparatus and training procedure might reveal different limits on performance. For example, it has been shown that AT is somewhat less pronounced when discrimination training is done in a WGTA than in a jumping stand (Dodwell, 1970). But what one can say on the basis of experiments using a single method is that certain discriminations are easier than others, or some cues are utilized more readily than others. It is in this more modest sense that we claim these experiments are relevant to understanding pattern recognition in the rat.

It would be of great interest to find other sets of patterns with which AT can be demonstrated, but so far they have proved to be elusive. To restrict the search within manageable proportions, a model for pattern coding seems to be prerequisite, but recently models for shape coding based on anything other than the Hubel and Wiesel receptive-field type of processing have not been in evidence. Other pattern sets which demonstrate AT might still be discoverable using a receptive field model, but none has so far occurred to us.

\section{REFERENCES}

Dodwell, P. C. Anomalous transfer after pattern discrimination training in rats and squirrels. Journal of Comparative and Physiological Psychology, 1970, 71, 42-51.

Dodwell, P. C., Ferguson, H. B., \& Niemi, R. R. Anomalous transfer in rats: Pattern element separation and discriminability. Bulletin of the Psychonomic Society, 1975, in press.

Niemi, R. R., \& DoDwell, P. C. The salience of two cues for pattern recognition in the rat. Canadian Journal of Psychology, $1971,25,375-383$.

(Received for publication October 20, 1975.) 